\title{
Design of NI sbRIO Based Working Fluid Level Detector and its Verification
}

\author{
Bingkun Gao ${ }^{1}$, Liyang $\mathrm{Jin}^{2}$ and Running $\mathrm{Gao}^{3}$ \\ ${ }^{1,2}$ Electrical Information \& Engineering Academy, Northeast Petroleum University \\ ${ }^{3} 180$ w. big springs rd. apt.23, Riverside, California, PC 92507 \\ 1'gbk@nepu.edu.cn, ${ }^{2}$ zhaoyanggolden@sina.com, ${ }^{3}\{269098201,57587416 @ q q . c o m\}$
}

\begin{abstract}
The depth of working fluid level of an oil well is one of the critical parameters that reveal the condition of down-hole environment. It should be measured periodically and accurately to stabilize the usual operation of pumping units. Acoustic method is currently the most common and most effective measurement method. This paper discuss a kind of working fluid level measuring equipment, which is based on NI single-board RIO embedded real-time processing system. By combing existing analysis method with wavelet transform theory, this device can automatically calculate the sound speed in wellbore annulus, and recognize the waveform reflected by fluid level. Results show that the device can effectively detect the depth of the working fluid level, meeting the needs of normal production activities in oil field.
\end{abstract}

Keywords: working fluid level, wavelet transform, acoustic method, NI single-board RIO

\section{Introduction}

In respects of oil field production control and equipment maintenance, the depth of working fluid level is one of the most important reference data. This parameter reveals the degree of pump shaft's submergence and the running power of pump, which should be adjusted timely during the usual operation on oil field. This adjustment is executed according to the variation of pressure in oil reservoirs and the change of reserves, so that the efficiency of well can be kept relatively stable. It not only ensures that the well daily output meets the demand, but also prevents pump machine from idling or overloading. Due to the different geographical environment and producing requirements, each well's down-hole environment has its own characteristics, which directly or indirectly affect the capability and accuracy of dynamic liquid level detection.

For existing devices that based on frequency division methods [1] or pressure change [2] which could not provide a satisfying rate of available measuring results, researchers have contributed a lot in the research of detecting working fluid level and designing automatic detection devices.

In terms of de-noising, the most known way is dual-channel analytical method, which divides signal into low frequency part--for sound speed calculation, and high frequency part-for reflect waveform detect [3]. However, this method could not effectively reflect the instantaneous characteristics of signal. A more effective way is spectral subtraction method, which supposes that the noise has a relative stable power spectrum characteristics so it can be subtracted without affecting the useful signal [4]; Many other theories have been raised to deal with de-noising procedure. For example, neural network algorithm has been used to enhance the amplitude of reflected wave and weaken noise [5]; wavelet transform theory for 
multi-resolution analysis is used to pick up wavelet coefficients of the useful signal and reconstruct the de-noised signal [6]; In terms of reflected waveform recognition, short time magnitude zero-crossing rate method, which is based on characteristic of sound source, makes the automatic reflected wave detecting more simple and relatively reliable [7]. In terms of sound velocity calculation, instead of the basic way of counting the reflected waveform caused by pup joint in a certain period time [8], Changsong Wang announced a calculation method based on AMDF theory, which is mainly used in estimating the pitch period of speech signal processing [9].

The author attempts to use NI company production: single-board RIO integrated embedded devices as the core of the signal processing unit, combining with LabVIEW software to design the device's hardware core unit for calculation and control. The device includes a LX45 FPGA control chip and a microprocessor chip, 16-bit A/D and $40 \mathrm{mHz}$ high frequency clock, $400 \mathrm{mHz} \mathrm{CPU}$, and $256 \mathrm{MB}$ RAM .It can be used to implement more complex signal processing method. By using the wavelet analysis method provided by the LabVIEW software on the device to deal with the noisy signal [10], this device succeeded in identifying the reflected waveform of dynamic liquid level in complex underground environment, and attained depth data of liquid level, which proved its effectiveness and the related algorithm.

\section{Measurement Theory and Hardware Design}

This device chooses the acoustic measurement method as the way to create sound wave pulse, which is currently widely used in detecting depth of liquid level. Acoustic reflection happens when encountered with obstacles and then return against the direction of the original transmission, acoustic measuring method in liquid level measurement do is based on this principle.

\subsection{Hardware Design}

The device usually contains an acoustic pulse generator, acoustic sensors, NI singleboard RIO device and related peripheral equipment, signal amplifier circuit, etc., as shown in Figure 1.

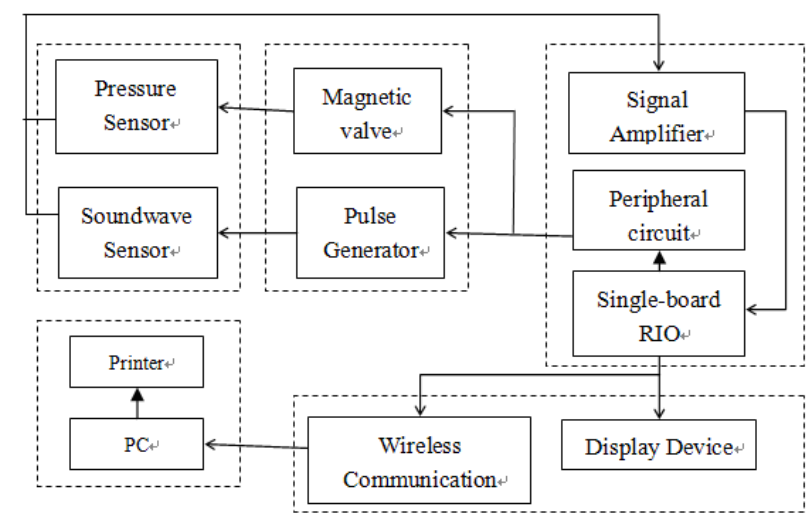

Figure 1. The Composition of Liquid Level Measuring Device

In the device, acoustic pulse generator contains a small air pump and a number of solenoid valves. When the internal pressure in the well rises to a specified value, the pump begins to work, providing the energy for the acoustic pulse. The single-board RIO 
equipment integrates the key functions such as device control, signal storage, signal amplification, de-noising, fluid level reflect signal recognition and data output, etc.

In order not to interfere with the normal work of the driving shaft, the device is usually installed at the interface of oil well casing annulus on the ground. When a sequence starts, single-board device send out control signals to open the magnetic valve and the acoustic wave generator in the device creates a sonic pulse which transmits through the well bore annulus flow downward. The sound wave reflects by the oil surface and then returns to the ground surface. The acoustic sensor begins to collect data at the time the magnetic valves begin to work. At the same time, signals are amplified and return back to single-board device. After analysis, result is shown on the display unit, and is sent via wireless transmission to the central computer in workstation. At last, the acoustic sensor installed on the ground receives the reflected signal. After amplification, noise filtering, the liquid level depth is identified by either artificial judgment or by automatic measurement device.

\subsection{Measurement Theory}

Down-hole environment of a typical oil well is shown in Figure 2.

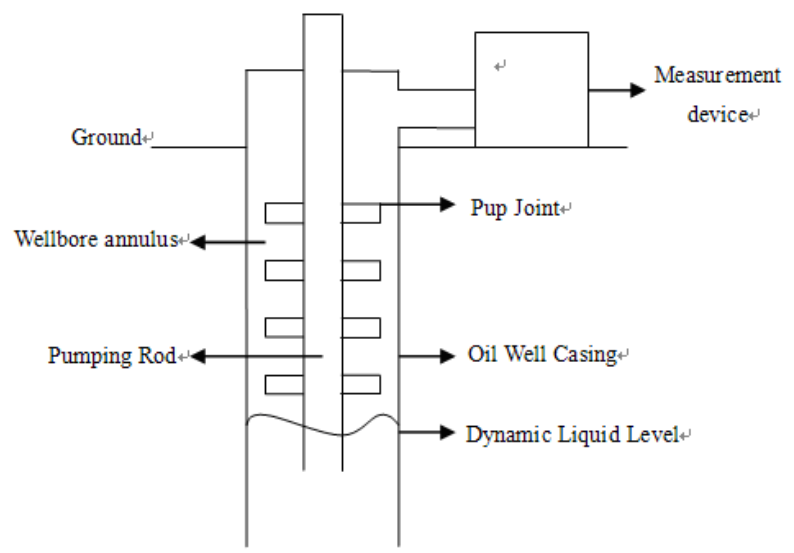

Figure 2. Downhole Environment of Oil Well

When travelling through the wellbore annulus downward, sound wave will encounter many pup joint fixed on the driving shaft, which can reflect part of the sound waves. Then sound wave reaches the working fluid level and produces a larger reflection, which returns from the bottom to the measuring device on the ground, accepted by the acoustic sensor. Usually, after amplification processing, the received acoustic signal will be filtered, and divided respectively into high frequency part and low frequency part, and being recorded separately at last. Sound wave will be attenuated in the process of transmission, and acoustic attenuation speed is relative with the frequency band of it: high frequency sound waves attenuate rapidly, so that the amplitude would decrease quickly with the increase of distance, so that the high frequency part of sound waves can only response several joint reflection waves near the ground; The low frequency part of acoustic attenuates relatively slow, therefore, low frequency part are more likely to record the reflected wave of liquid level. Reflection wave signal records the reflection waveform of dynamic liquid level and some other bigger obstacles, when the reflection to liquid surface occurs, a large amplitude change occurs. So it is relatively 
easy to identify the liquid level in the low frequency part, while the high frequency part is uesd to handle joint reflection waveform. Since the shaft is combined with many section of the rod of same length, so the pup joint spacing is equal. By calculating the average cycle of joint reflect wave, the average speed of sound waves in the wellbore could be calculated.

Suppose the length of pup joint is $L_{s}$, time spent for the sound waves pass through $\mathrm{N}$ joint is $T_{s}$, so the average sound velocity in the pipe can be calculated as follow way:

$$
V_{f}=\frac{2 N L_{s}}{T_{s}}
$$

Suppose the time spent from the magnetic valve opens to detect the liquid surface reflection wave is $T_{r}$, the dynamic liquid level depth of $\mathrm{S}$ is equal to:

$$
S=\frac{V_{f} T_{r}}{2}=\frac{N L_{s} T_{r}}{T_{s}}
$$

\section{Identification of Fluid Level}

Processing of reflected wave signal with a low-pass filter method can filter out part of noise in certain extent. However, well's condition differ in thousands ways: well path, frequency of rod stroke, pump operation conditions, pressure change in the annulus will influence the reflected wave signal in different degrees. Therefore, methods rely on Fast Fourier transform, which decomposite the signal by extracting and analyzing the fundamental frequency spectrum, can't meet the demand of calculating dynamic liquid level depth in the case of the complex underground environment.

Wavelet Transform is a new kind of signal analysis method developed on the basis of Short Time Fourier Transform (STFT). An integral wavelet transform of finite energy signal could be defined as follow:

$$
\left(w_{\psi} f\right)(b, a)=\frac{1}{\sqrt{a}} \int_{-\infty}^{+\infty} f(t) \psi\left(\frac{t-b}{a}\right) d t
$$

In this expression, $a>0$ stands for scale factor, $b$ stands for shift factor, and the wavelet function $\psi_{a, b}(t)=\frac{1}{\sqrt{a}} \psi\left(\frac{t-b}{a}\right)$ is stretched and scaled from original wavelet $\psi(t)$. The value of wavelet transforms reveals the time-frequency characteristics of signal in a local region related with a and b. Compared with Fast Fourier Transform (FFT) which focus on overall signal characteristics, Wavelet Transform pay more attention to the instantaneous changes of response signal, which therefore has more obvious advantage on the analysis of the instantaneous variation such as liquid surface reflection wave.

The parameters $\mathrm{a}$ and $\mathrm{b}$ are usually used in discrete form, where:

$$
a_{j}=2^{-j} \quad b_{j, k}=k / 2^{j} \quad j, k \in Z
$$

So the corresponding wavelet $\psi_{a_{j}, b_{j, k}}$ could be recorded as:

$$
\psi_{j, k}(t)=2^{j / 2} \psi\left(2^{j} t-k\right) \quad j, k \in Z
$$


While its wavelet transform is:

$$
\left(w_{\psi} f\right)\left(a_{j}, b_{j, k}\right)=<f, \psi_{j, k}>\quad j, k \in Z
$$

Instead of using these series expressions for practical application of the wavelet transform, problems are mostly be considered in the way of a multi-resolution analysis from $L^{2}(R)$ space. By using a fast algorithm of the discrete wavelet transform for numerical calculation, the calculation results could be analyzed after. The multi-resolution analysis refers to a group of nested closed subspaces, which satisfy several conditions:

a) $\quad \ldots \subset V_{-1} \subset V_{0} \subset V_{1} \subset \ldots$

b) $\overline{\bigcup_{j \in Z} V_{j}}=L^{2}(R)$

c) $\bigcap_{j \in Z} V_{j}=\{0\}$

d) $\quad f(\bullet) \in V_{j} \Leftrightarrow f(2 \bullet) \in V_{j+1}, j \in Z$

e) Exist a scale function $\varphi(x)$, make $\{\varphi(\bullet-k), k \in Z\}$ a set of orthogonal basis of $V_{0}$

If the relationship between the two parameters of scale functions $\varphi(x)$ satisfies the equation:

$$
\varphi(t)=\sum_{k \in Z} \sqrt{2} h_{k} \varphi(2 t-k)
$$

The function below could be proved to be an orthogonal wavelet:

$$
\psi(t)=\sum_{k \in Z} \sqrt{2}(-1)^{k} h_{1-k} \varphi(2 t-k)
$$

Which also means the wavelet group $\left\{\psi_{j, k}(t), j, k \in Z\right\}$ is one set of orthogonal basis of $L^{2}(R)$. Suppose:

$$
W_{j}=\operatorname{close}_{L^{2}(R)}\left(\psi_{j, k}(t), k \in Z\right) \quad j \in Z
$$

Then the equation below establishes:

$$
V_{j+1}=V_{j} \oplus W_{j} \quad j \in Z
$$

So we could get a corollary that:

$$
V_{j}=V_{j-1} \oplus W_{j-1}=V_{j-2} \oplus W_{j-2} \oplus W_{j-1}=\cdots=V_{m} \oplus W_{m} \oplus W_{m+1} \oplus \cdots \oplus W_{j-2} \oplus W_{j-1}
$$

In different scales, the useful signal and noise have different performance. By setting thresholds for different scale, the de-noising procedure could be achieved. This is the fundamental of detecting fluid level based on wavelet transform theory.

This paper chooses to use symlets4 wavelet during signal analysis. Sym4 wavelet is a kind of compactly supported, symmetric, biorthogonal wavelet, with high reduction of signal, and is more outstanding in detail extraction compared with the Daubechies wavelet. The development software LabVIEW attached with NI single-board RIO device provides symlets 
series wavelet function support, sym4 wavelet's basic characteristic curve in LabVIEW is shown in Figure 3 below.
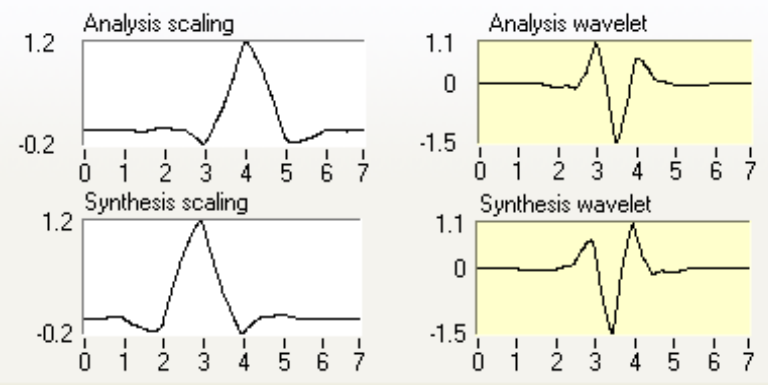

Figure 3. sym4 Wavelet Scale Function and Wavelet Function

In order to use wavelet transform to deal with the noise in signal, the signal should be divided in different scale at first, so the program can obtain the wavelet's coefficients and scaling factor. Then thresholds are set to filter out useless high frequency part. After this process, the signal could be reconfigurated. Screenshots of program made by using LabVIEW are shown in Figure 4:

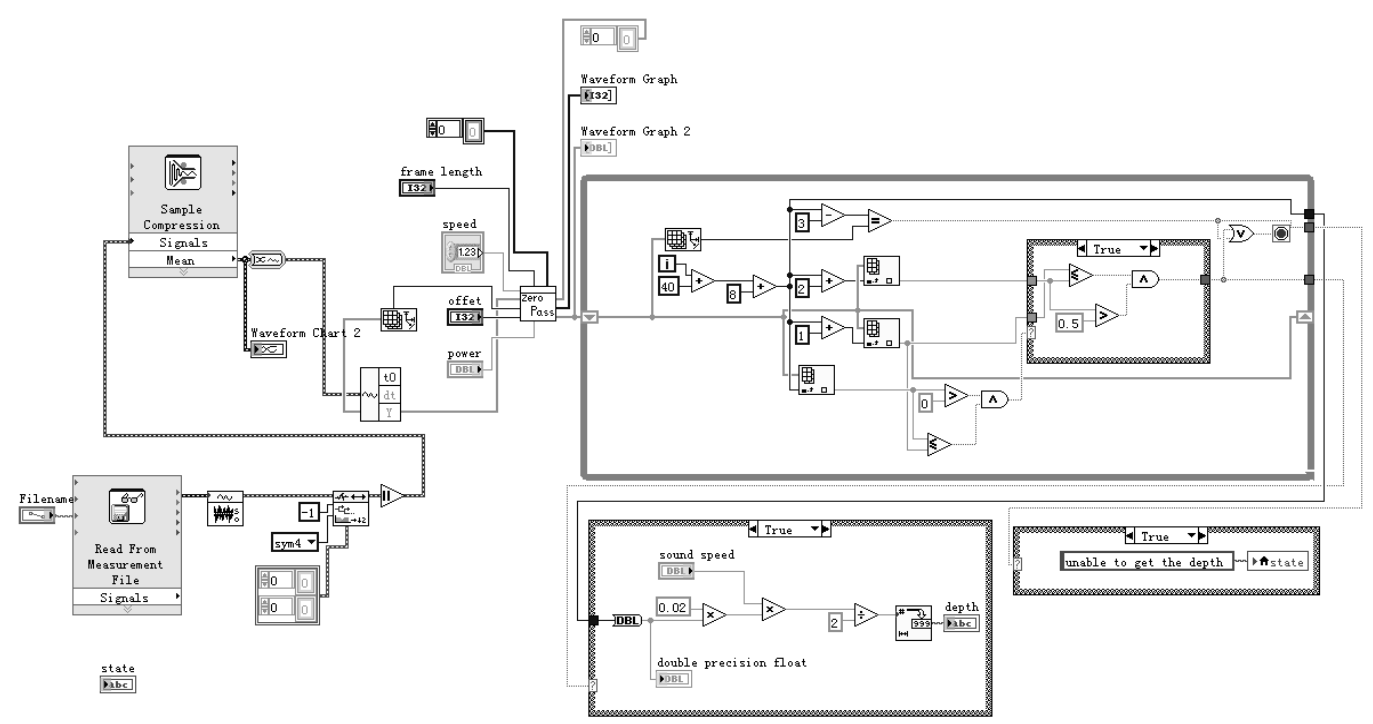

Figure 4. LabVIEW Program Sequence

\section{Field Test}

On finishing equipment debugging, the device is put into field test. Three oil wells in the second plant of Daqing oil field are chosen for the test, in which down-hole condition varies. The sample frequency is set at $1000 \mathrm{~Hz}$, and each sample continues for 10 seconds. Existing analysis method that uses dual channel digital filters is difficult to measure accurately the depth in these three well due to the lack of highlighting of the transient variation [11]. In the experiment, multi-resolution wavelet analysis tool in LabVIEW is used to decompose sample signal, in which sym 4 wavelet function is selected, and the decomposition layer is set as 4 . An ideal surface reflected wave signal is obtained by combining different layers with adjusted 
factors, as is shown in Figure 5. By comparing Figure 5-a. with Figure 5-b and 5-c, it can be seen that after sym4 wavelet decomposition, the reflected waveform is salient enough to identify.

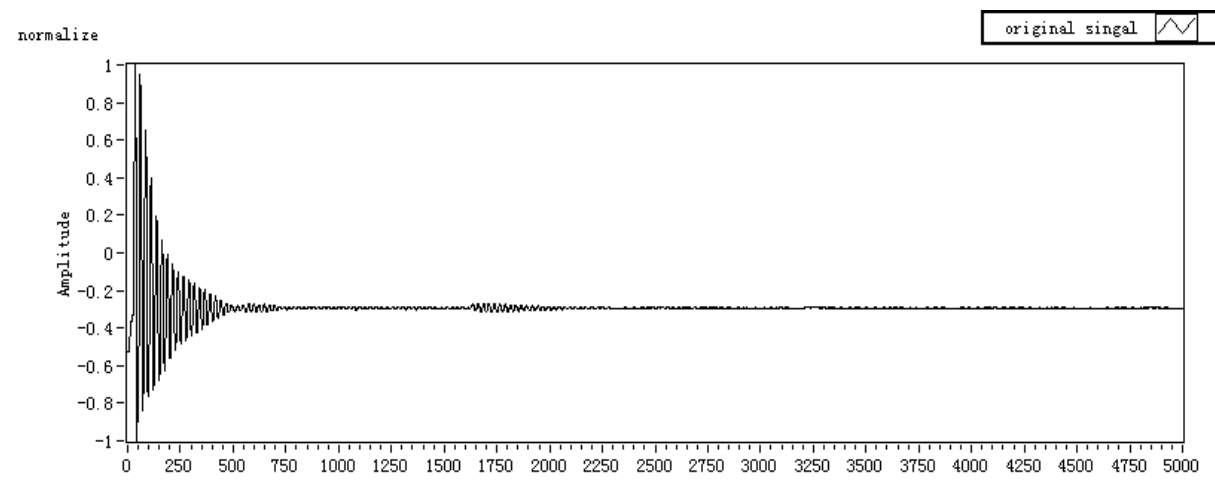

Figure 5-a. Origin Signal

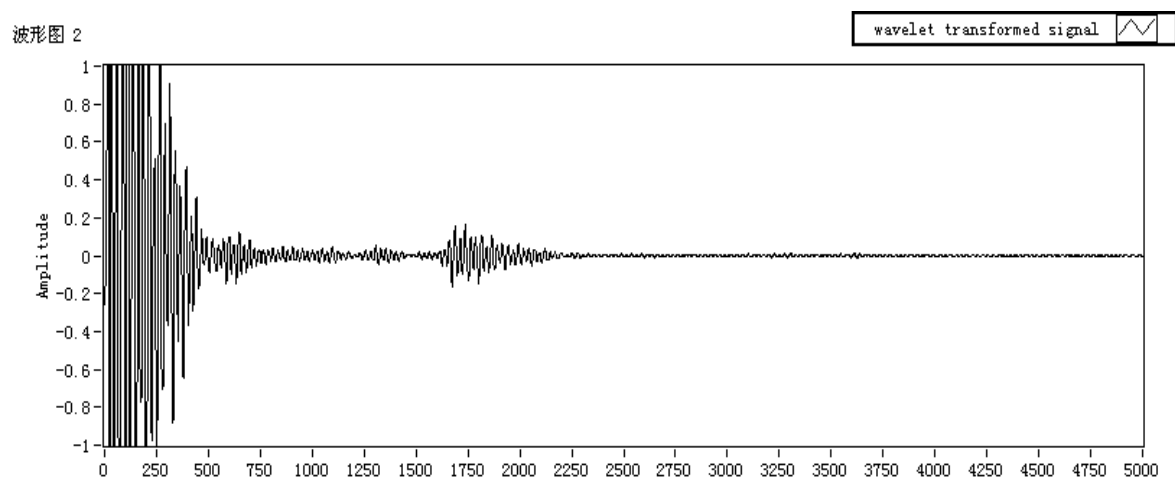

Figure 5-b. signal devided by 4 layer after sym4 wavelet decomposition

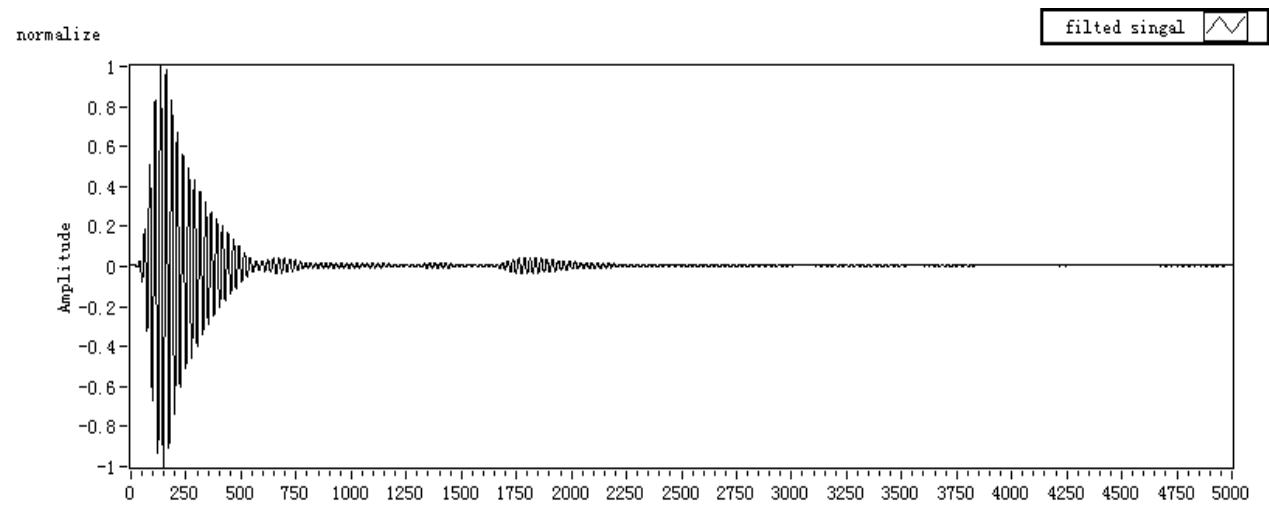

Figure 5-c. filtered signal of at the bandpass of $15 \mathrm{~Hz}$ to $25 \mathrm{~Hz}$

These three wells each has the problems of low borehole casing pressure, casing leaning and interference problem caused by pump machine, each of these well is tested tens of times for a more accurate result, and the test results are shown in the table 1: 


\section{Table 1. Field Test Data}

\begin{tabular}{|l|l|l|l|l|}
\hline \multirow{2}{*}{$\begin{array}{c}\text { Well } \\
\text { Number }\end{array}$} & \multicolumn{3}{|c|}{ Test data } & Field data \\
\cline { 2 - 5 } & $\begin{array}{c}\text { Calculated } \\
\text { sound speed }\end{array}$ & $\begin{array}{c}\text { Number } \\
\text { of test }\end{array}$ & $\begin{array}{c}\text { Average liquid } \\
\text { level depth }\end{array}$ & Real depth \\
\hline $712-\mathrm{P}-34$ & 420.18 & 15 & 343.12 & 342.08 \\
\hline $710-\mathrm{P}-51$ & 358.18 & 10 & 373.76 & 381.19 \\
\hline $710-\mathrm{P}-43$ & 396.26 & 10 & 651.90 & 660.57 \\
\hline
\end{tabular}

\section{Conclusion}

The site test result shows that the device designed for this test reaches the demand of practical application on oil field. Wavelet transform method is an effective way in eliminating noise and enhancing the reflected sound wave of working fluid surface. It also reveals better performance on picking up signal chrematistics than by dual channel filtering method. This method lowers the difficulty of identifying fluid level reflection signal, and achieves acceptable accuracy. It also has superiority in dealing with wells which have problems on identify the fluid level over traditional method.

\section{Acknowledgements}

This research is supported by "The Twelfth Five-Year" National Key Technology R\&D Program (Serial number: 2012BAH12B00) and Heilongjiang Province Key program of Ministry of education (Serial number: 12511z002)

\section{References}

[1] W. Jianxin, W. Changsong and Z. Jiaxin, "Echo method of increasing the measurement range and precision of determining the position of liquid surface in cased borehole", Petro Geology and Recovery Efficiency, vol. 16, (2009), pp. 93-96.

[2] G. Sam, M. M. Kaestenbauer, C. Burgstaller and E. Chevelcha, "Fully automated fluid level measurement tool", Society of Petroleum Engineers - SPE Asia Pacific Oil and Gas Conference and Exhibition, vol. 1, (2011), pp. 504-515.

[3] W. Zongrui, H. Xiangdong, H. Xiaohong and J. Ningbo, "Applications of digital filter inmeasuring oi1 wells", Chinese Journal of Scientific Instrument 2007 paper collection, vol. 2, (2007), March.

[4] L. Lixing and W. Haiwen, "Research on Signal Identification Technology in Liquid Level Detection of Oil Wells Using Acoustic method", China University of Petroleum, (2011), June.

[5] W. Xinjie and S. Dingyuan, "The Measurement of Well Liquid Level Based on Artificial Neural Networks", Journal Of Liaonig University, Natural Sciences Edition, vol. 27, (2000).

[6] W. Xinjie, S. Dingyuan and Y. Guanghui, "The Method for Processing Signals of Liquid level Depth of Oil's Well Based on Wavelet Transform”, Instrument Technique and Sensor, vol. 6, (1999) September, pp. 39-41.

[7] W. Xinjie, S. Dingyuan and W. Luchuan, "The Application of Fuzzy Sets Theory in Depth Measurement of an Oil Well's Level".

[8] Z. Jiaxin, W. Changsong, W. Jianxin and L. Zhimei, "Fluid Level Echo Recognition of Pumping Well Based on Short-time Magnitude Zero-crossing Rate Function”, Control and Instruments In Chemical Industry, vol. 34, (2007), pp. 59-62.

[9] R. Jian, W. Ping and Y. Tianjin, "A sonic velocity extracting method to dealwith dynamic working level echo signals in an oil well", Industrial Instrumentation and Automation, vol. 2008-5, pp. 63-65.

[10] Z. Jiaxin, W. Changsong, W. Jianxin, Y. Gengyun and L. Zhimei, "Calculation of Acoustic Velocity in Annular Space of the Pumping Well's Casing Based on AMDF", Chinese Journal of Sensors and Actuators, vol. 20, (2007) July, pp. 1648-1651.

[11] W. Haiwen and L. Lixing, "Signal denoising in liquid level detection with acoustic method using wavelet transform", Industrial Instrumentation \& Automation, vol. 2011-6, (2011) December, pp. 56-58. 


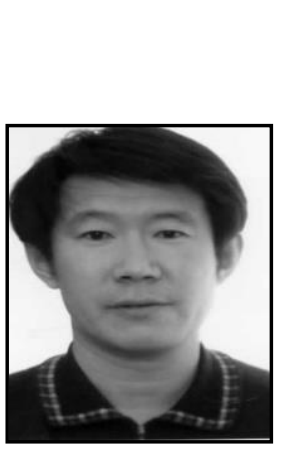

\begin{abstract}
Authors
Bingkun Gao, he received M.S degree and Doctor degree of Engineering in control theory and control engineering from Harbin Engineering University, People Republic of China in 1995 and 2003, and stood out from Northeastern University as postdoctor in Control Science and Engineering. He is currently the dean of Electrical and information engineering institute in Northeast Petroleum University. His currently research interests include Power system transmission control and fault diagnosis, multi-resource network monitoring and System simulation.
\end{abstract}

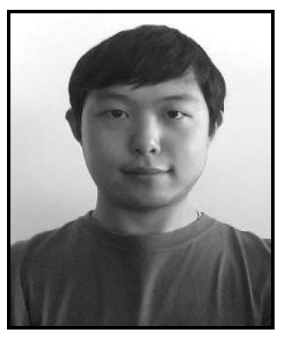

Liyang Jin, he received the Bachelor of Engineering degree in Information management and Information System from Beijing Language and Culture University in 2004, the People's Republic of China. Currently, he is the post graduate student of Electrical Information \& Engineering Academy in Northeast Petroleum University. His current research interests is automated signal processing.

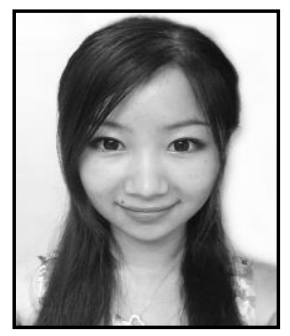

Running Gao, she received her master degree in Electronic Engineering from the University of California, the United State, in June 2013. She has the experience of doing research works in the Center for Environmental Research \& Technology. Her currently research interests are control \& robot and electrombile. 
International Journal of Signal Processing, Image Processing and Pattern Recognition Vol.6, No.6 (2013) 